Fig. 3. Schnitt aus einem decalcinirten Sternum. Schwache Vergrösserung. Die kleinsten vereinzelt liegenden Pigmentkörner sind bei dieser Vergrösserung nicht zu sehen, uur díe gehäuft liegenden Schollen treten hervor.

Fig. 4. Melanotisches, fettarmes Knochenmari aus dem Sternum; sehr dünner Schnitt. a Blutgefäss, zum Theil gefällt mit rothen und pigmenthaltigen weissen Blutzellen. b Fettzelle mit dem Stätzgewehe zusammenhängend. c Sternförmige Zelle pigmenthaltig. - In dem Gewebe sind pigmenhaltige Markzellen verschiedener Grösse zerstreut; einige von ihnen enthalten ausser dem feinkörnigen Pigment noch kreisrunde schwarzbraune Scheiben, die wobl als melanös entartete rothe Blutkörperchen aufzufassen sind.

\title{
XXVIII.
}

\section{Aphorismen zur pathologischen Anatomie der Centralorgane des Nervensystems.}

\author{
Von Dr. Rudolf Arndt, \\ professor in Greifswald.
}

1. Pigmentöse Degeneration der Markscheiden der Nervenfasern.

In den Intervertebralganglien eines 38jährigen Mannes, welcher an der tabischen Form der allgemeinen progressiven Paralyse gelitten und über drei Jahre ununterbrochen im Betle gelegen hatte, weil er durch so lange Zeit seine Beine nicht mehr zu brauchen im Stande gewesen war, fand ich eine Degeneration der Nervenfasern, welche bisher noch nicht beobachtet worden zu sein scheint. Das Mark einer grossen Anzahl der vorhandenen Fasern war nehmlich in ein braunes, körnig-krümliches, bei stärkeren Vergrösserungen öfters blättrig-schüllrigès Pigment umgewandelt worden und bedeckte als solches bald noch ziemlich fest, bald schon sehr lose die Axencylinder, welche an verschiedenen orten von ihrem Ursprung aus den sehr reich pigmentirten Ganglienkörpern weithin zu verfolgen waren.

Bei der Präparation war das Pigment, welches im Allgemeinen mit dem in den Ganglienkörpern gelegenen übereinstimmte, vielfach von dem Axencylinder abgestreift und aus den zerrissenen Schwann- 
schen Scheiden herausgespuilt worden. In grösseren oder kleineren Häufchen lag es nun zwischen den umgebenden Gebilden da und erinnerte unwillkürlich an die Pigmenthäufchen, welche mitunter im Gehirn und Rückenmarke angetroffen werden, wenn dieselben Sitz atrophischer Zustände sind.

Ich habe auf diese zoletzt erwähnten Pigmenthäufchen schon vor längerer Zeit ') besonders hingewiesen, um sie von den gewöhnlichen Körnchenzellen geschieden zu wissen, mit denen sie gelegentlich verwechselt werden können, und habe dabei geglaubt sie möglicherweise mit dem Pjgmente von centralen Ganglienkörpern, mit welchem ibre Elemente auch eine grosse Aebnlichkeit haben, in Zusammenbang bringen zu dürfen. Unmöglich ist durch die obige Beobachtung nicht geworden, dass ein solcher Zusammenhang thatsächlich bestebe und die fraglichen Pigmenthäuf́chen Trümmer untergegangener Ganglienkörper seien. Viel wahrscheinlicher indessen dürfte dennoch geworden sein, dass sie von zerfallenen Markscheiden herrührten, welche unter dem Einflusse atrophischer Zustände in derselben Weise degenerirten, wie die Fasern in den Intervertebral. ganglien unseres Tabikers. Die betreffenden Pigmenthäufchen oder Körnchenhaufen, wie ich sie erwähnten Ortes schlechtweg genannt habe, wären demzufolge im Gehirn und Riückenmarke eiı Zeichen von Atrophien der Nervenfasern, für welche, wie ich neuerdings ${ }^{2}$ ) gezeigt habe, die Veränderungen der Markscheiden bis jetzt das einzige sichere Kriterium sind.

2. Tubulöse Degeneration der Markscheiden der Nervenfasern.

In Erweichungsheerden des Rückenmarkes oder Stellen desselben, welche der grauen Degeneration verfallen waren, begegnete ich öfters Nervenfasern, deren Markscheiden eine eigenthümliche Abweichung von der Norm erkennen liessen. Sie waren sebr stark aber ungleichmässig gequollen und darum auffällig varicos, sodann von einer Menge ungleichmässig breiter und unregelmässig verlaufender dunkler Längsstreifen durchzogen und deshalb wieder wie zerschlissen. Bei genauerer Untersuchung aber ergab sich, dass Letzteres nur auf einer optischen Täuschung beruhte und davon

1) Arch. f. Psychiatr. u. Nervenkrkht. Bd. II. S. 775.

2) Dieses Archiv Bd,ILX, S. 511 a. ff. 
herrührte, dass die sonst solid erseheinenden Markscheiden sich in eine Anzahl von Röhren zerlegt hatten, welche durch bald schmalere, bald breitere Zwischenräume getrennt, eine in die andere gesteckt, hülsenartig den Axencylinder umgaben.

Weun man Querschnitle eines in chromsauren Salzen gehärteten Rückenmarkes oder auch peripherischen Nervenstammes mit Carmin färbt und danach in Canadabalsam, Damarlack oder sonst dergleichen einschliesst und dann der mikroskopischen Besichtigung unterwirft, so gewahrt man, dass durch Carmin sich nur die die einzelnen Nervenfasern trennenden Bindegewebssepta und in ihnen die Axencylinder gefärbt haben. Ihr Mark hat die in der Härtungsflüssigkeit erworbene gelbliche Färbung beibehallen. Sieht man aber genauer $\mathrm{zu}$, so entdeckt man in ihm, das als eine rundliche, elliptische, ovale Scheibe erscheint, in deren Mitte den rothen Axencylinder und um diesen herum in unregelmässigen Abständen feine concentrische rothe Linien, welche die Markscheibe in ebenso viele unregelmässig concentrische Ringe theilen.

Wodurch diese concentrischen rothen Linien bedingt werden, ist noch umbekannt. Offenbar müssen sie aber doch von Stoffen herrühren, welche die Markscheiden in concentrischen Touren, beziehungsweise als concentrische Röhren durchsetzen. Das eigentliche Mark der Scheiden muss danach aber auch einen gegliederten Bau haben und ebenfalls aus concentrischen Röhren bestehen, welche durch eine differente Masse von einander getrennt sind.

Wie weit diese Verhältnisse schon in Leben bestehen, wie weit sie erst nach dem Tode zur Ausbildung, vielleicht auch erst Entwicklung ïberhaupt kommen, ist bis jetzt unaufgehellt. Die oben erwähnte Anomalie der Markscheiden hängt indessen mit ihnen auf das innigste zusammen. Sie kommt dadurch zu Stande, dass die Interstitien zwischen den Markscheidenröhren, die wir als mit irgend welchem Stoffe, der durch Carmin sich roth färbt, ausgefüllt annehmen, und die für gewöhnlich nur äusserst schmal und darum kaum sichtbar sind, dass diese sich erweitern und za unverhältnissmässig breiten Räumen ausdehnen. Die einzelnen Röhren der Markscheiden werden dadurch beträchtlich von einander entfernt, stehen bald mehr, bald weniger weit, von einander ab und bieten dadurch das mikroskopische Bild dar, welches ich von ihnen zu entwerfen gesucht habe. 
Wie dem endgültig nun aber auch sei, die beschricbene Anomalie scheint nichtsdestoweniger' mit grosser Bestimmtheit dafür zu sprechen, dass den Markscheiden der Nervenfasern vielfach ein röhrenförmiger Bau zukomme, der schon während des Lebens besteht und durch die Wachsthumsverhältnisse der Markscheiden, durch schichtenweise Auflagerung von aussen her, wie ich anderen Orts noch einmal zeigen werde, verursacht wird.

\section{Spaltung des Axencylinders.}

In. den Spinalganglien des in 1 erwähnten 38 jährigen Paralytikers, sowie in den Ganglia Gasseri eines Anderen, bei dem indessen keine tabischen Erscheinungen bestanden hatten, fand ich Nervenfasern vor, welche vielfach ihres Markes beraubt, also atrophisch waren, und Veränderungen der Axencylinder erfahren hatten, welche bisher nur sehr vereinzelt und unter ganz besonderen Umständen an peripherischen Nerven beobachtet worden sind.

Die Veränderungen betrafen immer sehr breite Fasern und bestanden darin, dass die ebenfalls sehr breiten Axencylinder etwas glänzender als gewöhnlich geworden und von einer Anzahl differenter Streifen, zwischen denen Punktreihen eingeschaltet waren, der Länge nach durchzogen wurden. In Carminpräparaten erschienen diese veränderten Axencylinder blass perlgrau und die Streifen darin deutlich rosaroth, die Punktreihen schwach röthlich. Die Anzah! der Streifen, welche bei einer Vergrösserung von circa $800 \mathrm{mal}$ als deutliche Bänder erschienen, welche bald mehr, bald weniger parallel neben einander hinzogen, wechselte zwischen drei bis füf.

lch bin geneigt diese Veränderungen mit einer Spaltung des Axencylinders in seine Primitivfibrillen in Zusammenhang zu bringen und damit die Affection als ein Analogon der von $\mathrm{Remak}^{2}$ ), Neumann ${ }^{2}$ ), Eichhorst ${ }^{3}$ ) und Westphal ${ }^{4}$ ) nach Bleilahhmung im $\mathrm{N}$. radialis beobachteten Vorgänge anzuseben.

Wie erwähnt sah jch diese Spaltung nur an sehr breiten Axencylindern, wie sie nur selten, um nicht zu sagen ausnahmsweise, zu Gesicht kommen. In Anbetracht des ungewöhnlichen Glanzes

1) Dieses Archiv Bd. XXIII. S. 441.

2) Archiv f. Heilkunde. Jahrg. 9. S. 193.

3) Dieses Archiv Bd. ILX. S. 1.

) Archiv für Psychiatrie Bd. IV. S. 776. 
den sie hatten, in Anbetracht der hreiten Fibrillen, die sie bargen, und die doch normaler Weise nur sehr fein sind, glaube ich indessen nicht, dass diese Breite ihnen von Hause aus zukam, sondern dass sie dieselbe erst im Laufe der Krankheit erlangt hatten, dass sie hypertrophisch waren, ohne freilich dabei varicos zu scin, wie das in solehem Zustande meistens der Fall zu sein pflegt. Auffallend musste nur dabei sein, dass diese Hypertrophien der Axencylinder sich herausgebildet haben sollten, während gleichzeitig in anderen Theilen der Nerv atrophisch zu Grunde ging. Ich habe aber gezeigt ${ }^{1}$ ), dass nicht alle anscheinend hypertrophischen Axencylinder auch wirklich hypertropbisch sind, sondern dass ibre Volumzunahme auch noch aus anderen Gründen als einer Hypertrophie erfolgt sein könne. Ich habe gezeigt, dass eine einfache Infiltration des Axencylinders mit fremden Stoffen, also vielleicht auch mit dem zerfallenden Mark seiner Scheiden dies bewerkstelligen könne, und dass somit die anscheinende Hypertrophie nichts Anderes als eine einfache Quellung zu sein brauche, die um so eher eintreten werde, je weiter der Nerv schon in seiner Emährung heruntergekommen sei. Eine einfache Quellung konnte demnach auch in den uns augenblicklich beschäftigenden Fällen vorliegen und die scheinbare Hypertrophie der Axencylinder bei gleichzeitiger Atrophie der Markscheiden der betreffenden Fasern vortäuschen.

Die Spaltung des Axencylinders würde demgemäss in Zusammenhang stehen mit einer Beeinträchtignng in ihrer Ernährung, mit einer Atrophie, der sie anheim gefallen. Und wenn nach den an peripherischen Nerven gemachten Beobachtungen sie mit der Regeneration durchschnittener oder gelähmter Nerven in Beziehung stehen soll, so ist das dieser Annahme doch keineswegs entgegen, weil die bezügliche Spaltung, wie das namentlich aus den Untersuchungen Eichhorst's hervorzugehen scheint, viel früher eintritt, als die Regeneration selbst, und diese darum in der eigenthümlichen Weise sich macht, weil durch die Atrophie der verletzten oder peripherischen Nerven erst die Bedingungen dazu geschaffen wurden.

\section{Kerntragende Nerrenfasern.}

Wiederholt habe ich in der lelzten Zeit im Gehirn und Rückenmarke des Menschen Nervenfasern gefunden, welche mit blassen,

) Archiv für Psychiatrie Bd. II. S. 766. 
länglich-ovalen Kernen besetzl waren. Die Kerne bestanden aus einer mattglänzenden, leichtkörnigen Masse, in welche 2-3 grössere Körnchen als Kernkörperchen eingestreut waren, und sassen wunderbarer Weise nicht der Oberfläche der Fasern auf, soudern den Axencylindern. Es waren also Nervenfasern mit kerntragenden Axencylindern, die ich gefunden hatte.

Gelegentlich habe solche kerntragenden Axencylinder icb auch in den Spinalganglien des Menschen angetroffen und zwar nicht blos in den eigentlichen Spinalganglien, den Intervertebralganglien, sondern auch im Ganglion Gasseri und Jugulare vagi.

Einunal auf die Sache aufmerksam geworden, stellte ich weitere Nachforschungen an, konnte aber lange Zeit zu keinen bestimmten Resultaten gelangen. Beim Menschen kommen die kerntragenden Axencylinder in den Centralorganen jedenfalls nicht häufig vor und scheinen mebr der Ausdrack individueller Verbältnisse $z u$ sein als typisch und an bestimmie Localitäten gebunden. Oefter stösst man auf sie bei Thieren und sah ich sie z. B. mebrlach in der Trabs cerebri der Katze, im Cornu Ammonis und Bulbus olfactorius des Kaninchens, im Cerebellum und Lobus temporalis des Hundes.

Da nun diese Gebilde, obgleich sie dem Angegebenen nach immerhin in ziemlicher Verbreitung vorkommen, dennoch nicht etwas Gewöhnliches sind, sondern etwas mehr oder weniger Ausnahmsweises, von der Norm Abweichendes, so drängt sich die Frage auf, was sie wohl eigentlich seien und was sie wohl auch zu bedeuten haben.

Um diese frage zu erledigen ist es nothwendig einen Blick auf die Entwickelung des centralen Nervensystems zu werfen. Die gesammten Elemente desselben gehen aus Zellen mit runden Kernen hervor, natürlich also auch die Nervenfasern. Es muss dies aber noch besonders betont werden, da in neverer Zeit mit grosser Bestimmtheit behauptet worden ist, dass nachweislich das nicht der Fall wäre und die Nervenfasern nur won Zellen mit ovalen Kernen abstammten. Ich muss nach Allem, was ich bisher gesehen habe, dem auf das Bestimmteste widersprechen, muss aber zugeben, dass während der Entwickelung der Nervenfasern, d. h. während des Auswachsens des Protoplasmas der rundkernigen Bildungszellen al späteren Nervenfasern sich verhältnissmässig grosse ovale kernähnliche Körper bilden, welche den angelegten Fasern anhaften und zu der Täuschung Veranlassung geben, als ob die sich bildender! 


\section{4}

Fasern mit ovalen Kernen besetzt seien und von Zellen mit derartigen Kernen abstammen.

Der Vorgang, der beim Hühnchen beobachtet worden ist, ist folgender:

Die rundkernigen Bildungszellen, welche nur mit einem sehr spärlichen Protoplasma ausgestattet sind, umgeben sich zu einer gewissen Zeit mit einem mächtigeren. Dabei erfäbrt dasselbe gewisse Differenzirungen und zeigt namentlich das Auftreten von kleinen glänzenden Kügelchen mit einem differenten Inhaltskőrper, welche in die glasig-gallertige Grundsubstanz eingebettet sind. Bei vielen Zellen sondern sich nun während dieses Differenzirungsprozesses grössere und kleinere, scharf umschriebene länglich-ovale Körperchen aus, welche Anfangs der Grundsubstanz noch vollständig gleichen, dann etwas homogener werden, ein, meistens jedoch mehrere dunklere Kügelchen in ihrem Inneren erhalten und damit das Aussehen von Kernen gewinnen. Es sind dies aber nichts Anderes als Verdichtungen des normalen Protoplasma, Knoten desselben, entstanden um Atractionspunlste, die sich aus irgend welchem Grunde in seiner Masse gebildet batten.

Zwischen diesen Knoten des Protoplasma und dem Kerne seiner Zelle geht das Hauptwachsthum der letzteren selbst ror sich. Bei den Nervenbildungszellen tritt hier die Protoplasmazunahme ganz besonders hervor. Während die Zelle, um mich so auszudrücken, um den Kern herum so gut als gar nicht wächst, wächst sie zwischen demselben und dem neu entstandenen Protoplasmaknolen mächtig aus, verlängert sich fadenförmỉg und schiebt dadurch den Protoplasmaknoten von seiner ursprünglichen Bildungsstätte weit weg. Nachdem das geschehen, bildet sich in der Nähe des Kernes wieder ein Protoplasmaknoten. Derselbe wird wieder weggeschoben. Danach bildet sich ein dritter, vielleicht auch noch ein vierter Protoplasmaknoten, die wieder weggeschoben werden, und so entsteht ein langer Protoplasmafaden, der oberflächlich besehen von Zeit zu Zeit leichte Anschwellungen oder Varicositäten zeigt, bei genauerer Besichtigung aber mit kleinen länglichen Kernen besetzt ist, und der der rundkernigen Bildungszelle nur wie zufällig anliegt.

Die Bildungszelle sendet indessen nicht blos nach einer Richtung eine derartige Faser aus, sondern auch nach der entgegen- 
gesetzten, so dass zu einer gewissen Zeit sie in Mitten einer mit kleinen ovalen Kernen besetzten Faser zu liegen scheint. Die Faser nimmt an Masse zu, verbindet sich mit anderen, in gleicher Weise entstandenen Fasern und differenzirt ihre späteren Bestandiheile, zunächst allerdings blos den Axencylinder und seine Fibrillen. Dabei verändern sich aber die sie bedeckenden kleinen Kerne, die ursprünglichen Protoplasmaknoten. Sie vergrössern sich, werden homogener, scbnüren sich noch mehr von ihrer Umgebung ab und sondern mehrere grössere differente Körperchen in sich aus, von denen eins oder zwei häufig durch ihre Grösse auffallen, und die sich zu ihnen wie Kernkörperchen zta einem Kern verhalten. Und während alles Dieses vor sich geht, löst sich die so gebildete Nervenfaser, noch blos ein nackter Axencylinder, von ihrer resp. von ihren Bildungszellen $\mathrm{ab}$ und lässt diese oder auch blos thre Kerne seitlich liegen. Wir haben dann einen Axencylinder vor uns, welcher in keinem Zusammenhange mehr mit rundkernigen Zellen steht und ganz allein aus langkernigen, deren Kerne inn noch bedecken, hervorgegangen zu sein scheint. Mancherorts z. B. in den Wurzeln der spinalen Nerven geht dieser Prozess mit einer so riesigen Schnelligkeit vor sich, dass er in kaum 24 Stunden vollendet erscheint und nicht zu verstehen wäre, wenn nicht Analoga aus anderen Partien, namentlich des Gehirns, wo die Entwickelung langsamer sich vollzieht, über jhn Aufsehluss gäben.

Den Nervenfasern ganz ähnlich verläuft die Bildung der ersten Ganglienkörper. Auch sie gehen aus rundkernigen Bildungszellen hervor, in welchen Verdichtungen des Protoplasma sich machen, ehe es zum Auswachsen der Fortsätze kommt, und bei denen darnach die Fortsätze mit den aus den Protoplasmaverdichtungen hervorgegangenen ovalen Knoten wie mil Kernen bedeckt sind.

Normaler Weise schwinden in späterer Zeit wieder diese PseudoKerne. Wann? Das weiss ich nicht zu sagen. Die Axencylinder umgeben sich mit Mark; die Ganglienkörper und ihre Fortsätze festigen sich, spalten sich in eine Anzabl fibrillenäbnliche Streifen, doch ohne gerade in solche zu zerfallen; von den pseudokernen indessen bleibt so gut als Nichts übrig. Nur hin und wieder, jedoch wie es scheint, noch immer mit einer gewissen Regelmässig keit findet man Etwas von ibnen bei Thieren, nur ausnahmsweise und allem Anscheine nach unter ganz besonderen Verhältnissen, 
beim Menschen. Was wird aus ibnen? Ich weiss es nicht. Vielleicht gehen sie als Körnchenzellen zu Grunde, welche ja in einer gewissen Periode der Entwickelung des Nervensystems eine auffallende Rolle spielen. Genug, normaler Weise verschwinden sie und namentlich im Nenschen ohne irgend eine Spur zu hinterlassen. Wenn sie das nun nicht thun, was folgt daraus? Dass die Entwickelung der Fasern, an welchen sie sich finden, nicht die Höhe erreicht hat, welche sie erreichen soliten, und wenn viele solche Fasern, vornehmlich in bestimmten Gebieten des Gehirns und Rückenmarkes vorhauden sind, dass diese Gebiete in toto auch nicht die Entwickelungsstufe erreicht haben, zu welcher sie gelangen soliten. Die Anwesenheit der Pseudokerne zeigt also eine Entwickelungshemmung der bezüglichen Axencylinder resp. Nervenfasern an, ein Verbarren derselben auf einem dem embryonalen Zustande näheren, und sind die kerntragenden Axencylinder in grösserer Zahl vorhanden, so eine Entwickelungshemmung des Gohirns und Riickenmarkes resp. der Theile desselben, in welchen sie sich finden.

Ich habe die kerntragenden Nervenfasern des Gehirns und Rückenmarkes bisher nur bei geisteskrank verstorbenen Individuen gefunden, die kerntragenden Nervenfasern der Spinalganglien bei paralytisch zu Grunde gegangenen Personen. Wenn sie nun wirklich das sind, was sie sein sollen, Zeugen einer niedrigeren Entwickelung, einer Bildungshemmung, waren sie da vielleicht in den betreffenden Fällen Fingerzeige, warum etwa die Individuen geistes. krank wurden und paralytisch zu Grunde gingen, $d$, h. gaben sie vielleicht die Erklarung für die besondere Disposition $a b$, welche jenen Individuen einmal zu geistiger Erkrankung, das andere Mal zu paralytischen Zuständen inne wohnte? -. Ich habe grosse Neigung das anzunehmen and bin uberhaupt gewillt, seit ich die ganze Angelegenheit kennen lernte, das Wesen der angebornen neuropathischen und psychopathischen Diathese vorzngsweise in Bildungshemmungen $z u$ suchen, welche die feinsten Elemente des Nervensystems aus diesem oder jenem Grunde erfahren haben, und̀ die sich unter Anderem vorzugsweise durch das Uebrigbleiben transitorischer Bildungen aus der Entwickelungsperiode kenntlich machen. 